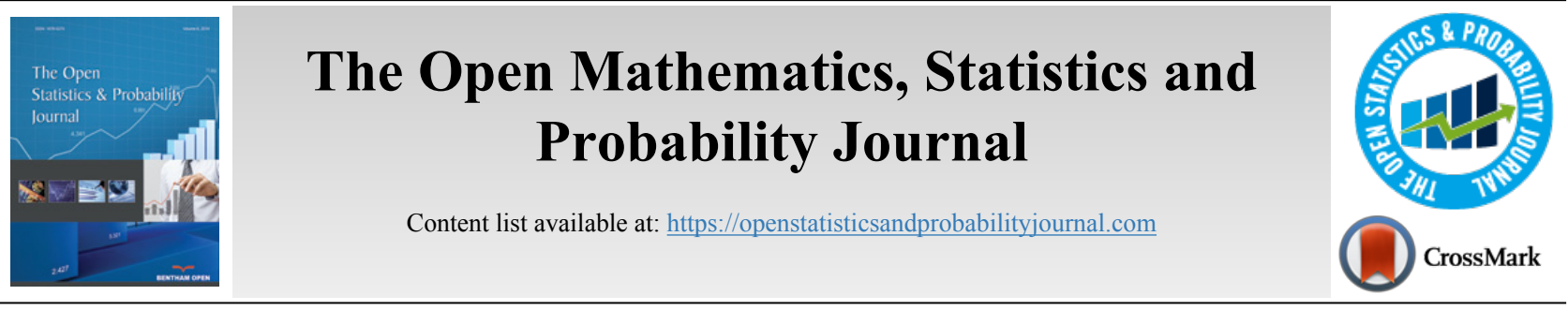

RESEARCH ARTICLE

\title{
On The Distribution of Partial Sums of Randomly Weighted Powers of Uniform Spacings
}

Emad-Eldin A. A. Aly ${ }^{1, *}$

${ }^{1}$ Department of Statistics \& Operations Research, Faculty of Science, Kuwait University, Safat 13060, Kuwait

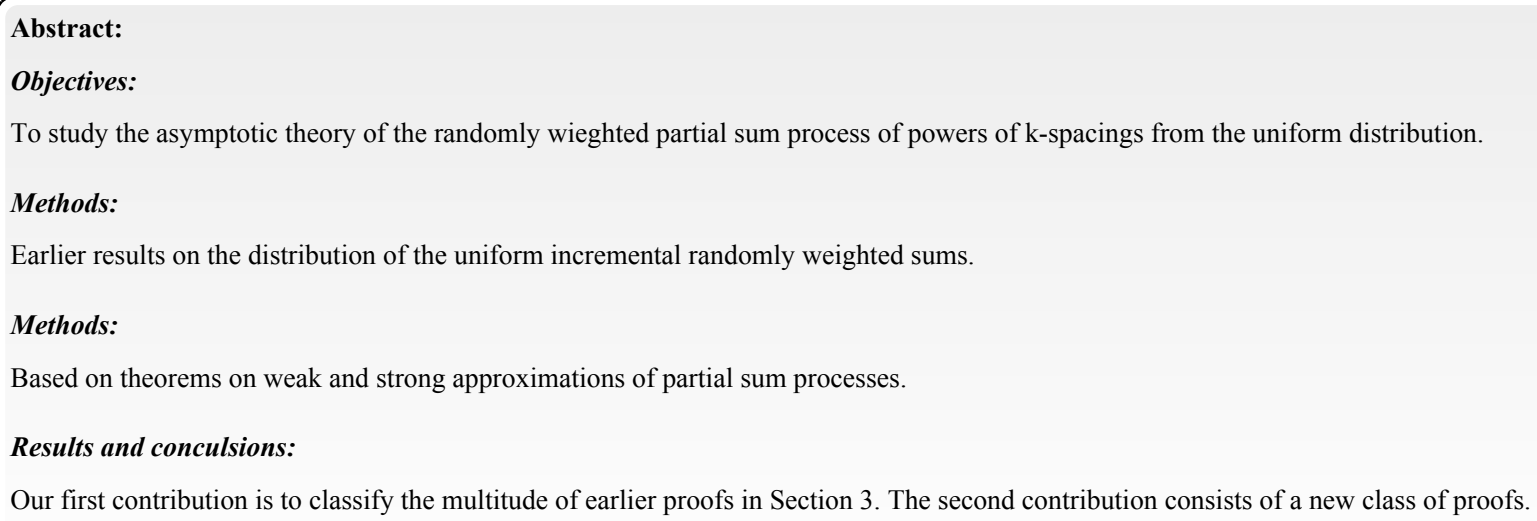

Keywords: Uniform spacings, Weak convergence, Gaussian process, Incremental asymptotic convergence, Random Sample, k spacings.

\begin{tabular}{|l|l|l|l}
\hline Article History & Received: February 02, 2019 & Revised: November 06, 2019 & Accepted: November 16, 2019
\end{tabular}

\section{INTRODUCTION}

Let $0=U_{(0)} \leq U_{(1)} \leq U_{(2)} \leq \cdots \leq U_{(n-1)} \leq U_{(n)}=1$ be the order statistics of a random sample of size $(n-1)$ from the $U(0,1)$ distribution. Let $k=1,2, \ldots$ be arbitrary but fixed and assume that $n=m k$. The $U(0,1) \mathrm{k}$-spacings are defined as

$$
R_{i, k}=U_{(i k)}-U_{((i-1) k)}, i=1,2, \cdots, m .
$$

Let $X_{1}, X_{2}, \ldots$ be iidrv with $E\left(X_{\mathrm{i}}\right)=\mu, \operatorname{Var}\left(X_{\mathrm{i}}\right)=\dot{o}^{2}<\infty$ and common distribution function $\mathrm{F}($.$) . Assume that the Xi's are$ independent of the Ui's. Define

$$
S_{m}(t, k, r, F)= \begin{cases}\sum_{i=1}^{[m t]} R_{i, k}^{r} X_{i}, & \frac{1}{m} \leq t \leq 1 \\ 0, & 0 \leq t<\frac{1}{m}\end{cases}
$$

where [s] is the integer part of $\mathrm{s}$ and $r>0$ is fixed.

Looking at $S_{m}(t, k, r, F)$ of (2) as a weighted partial sum of

* Address correspondence to this author at Department of Statistics \& Operations Research Faculty of Science, Kuwait University, Safat 13060, Kuwait; Tel: +965 66655671; E-mail: eealy50@gmail.com the $X^{\prime} s$, Van Assche [1] obtained the exact distribution of $S_{2}(1$, $1,1, F)$. Johnson and Kotz [2] studied some generalizations of Van Assche results. Soltani and Homei [3] considered the finite sample distribution of $S_{n}(1,1,1, F)$. Soltani and Roozegar [4] considered the finite sample distribution of a case similar to $S_{m}$ $(1, k, 1, F)$ in which the spacings (1) are not equally spaced. It is interesting to note that $S_{m}(t, k, r, F)$ of (2) is also a randomly weighted partial sum of powers of k-spacings from the $U(0,1)$ distribution.

Here, we will obtain the asymptotic distribution of the stochastic process

$$
\begin{aligned}
& \alpha_{m}(t, k, r, F)=\left\{\begin{array}{l}
m^{\frac{1}{2}}\left\{k^{r} m^{r-1} S_{m}(t, k, r, F)\right. \\
0,
\end{array}\right. \\
& \left.-t \mu \mu_{r, k}\right\}, \frac{1}{m} \leq t \leq 1 \\
& 0 \leq t<\frac{1}{m} \text {, }
\end{aligned}
$$




$$
\mu_{l, k}=\frac{\Gamma(k+l)}{\Gamma(k)}, k \geq 1 l>0
$$

and $\Gamma($.$) is the gamma function.$

The motivations and justifications of this work are given next. First, as noted by Johnson and $\operatorname{Kotz}[2], S_{2}(1,1,1, F)$ is a random mixture of distributions and as such it has numerous applications in Sociology and in Biology. Second, the asymptotic theory of $S_{m}(t, k, r, F)$ is a generalization of important results of Kimball [5], Darling [6], LeCam [7], Sethuraman and Rao [8], Koziol [9], Aly [10] and Aly [11] for sums of powers of spacings from the $U(0,1)$ distribution. Finally, we solve the open problem of proving the asymptotic normality of $S_{m}(1, k, 1, F)$ proposed by Soltani and Roozegar [4].

\section{METHODS}

\subsection{The asymptotic distribution of $\alpha \mathrm{m}(., k, r, F)$}

Let $Y_{1}, Y_{2}, \ldots$ be iidrv with the exponential distribution with mean 1 which are independent of the $X i$ 's. By Proposition 13.15 of Breiman [12] we have for each $n$,

$$
\begin{aligned}
& \left\{0=U_{(0)}, U_{(1)}, U_{(2)}, \cdots, U_{(n-1)}, U_{(n)}=1\right\} \\
& \stackrel{D}{=}\left\{0, \frac{Y_{1}}{\sum_{i=1}^{n} Y_{i}}, \frac{Y_{1}+Y_{2}}{\sum_{i=1}^{n} Y_{i}}, \cdots, \frac{Y_{1}+Y_{2}+\cdots+Y_{n-1}}{\sum_{i=1}^{n} Y_{i}}, 1\right\} .
\end{aligned}
$$

Hence, for each $m$,

$$
\left\{R_{i, k}, 1 \leq i \leq m\right\} \stackrel{D}{=}\left\{\frac{z_{i, k}}{\sum_{i=1}^{m} Z_{i, k}}, 1 \leq i \leq m\right\},
$$

where for $1 \leq i \leq m$,

$$
Z_{i, k}=Y_{(i-1) k+1}+\cdots+Y_{i k}
$$

are iid $\operatorname{Gamma}(k, 1)$ random variables. Hence, for each $m$

$S_{m}(t, k, r, F) \stackrel{D}{=} \sum_{i=1}^{[m t]} Z_{i, k}^{r} X_{i} /\left(\sum_{i=1}^{m} Z_{i, k}\right)^{r}, 0 \leq t \leq 1$

and

$$
\alpha_{m}(\cdot, k, r, F) \stackrel{D}{=} \beta_{m}(\cdot, k, r, F)
$$

where

$$
\beta_{m}(t, k, r, F)=\left\{\begin{array}{l}
0, \quad 0 \leq t<\frac{1}{m} . \\
m^{\frac{1}{2}}\left\{k^{r} m^{r-1} \sum_{i=1}^{[m t]} Z_{i, k}^{r} X_{i}\right. \\
\left./\left(\sum_{i=1}^{m} Z_{i, k}\right)^{r}-t \mu \mu_{r, k}\right\}, \quad \frac{1}{m} \leq t \leq 1
\end{array}\right.
$$

Let $\mu_{1, \mathrm{k}}$ be as in (4). Note that

$$
\begin{gathered}
E\left(Z_{i, k}^{l}\right)=\mu_{l, k}, \\
E\left(Z_{i, k}^{r} X_{i}\right)=\mu \mu_{r, k}, \\
\sigma_{r, k}^{2}=\operatorname{Var}\left(Z_{i, k}^{r} X_{i}\right)=\sigma^{2} \mu_{2 r, k}+\mu^{2}\left\{\mu_{2 r, k}-\mu_{r, k}^{2}\right\}
\end{gathered}
$$

and

$$
\operatorname{Cov}\left(Z_{i, k}^{r} X_{i}, Z_{i, k}\right)=r \mu \mu_{r, k}
$$

The following Theorem will be needed in the sequel.

Theorem A. There exists a probability space on which a two-dimensional Wiener process $\left\{\underline{\mathbf{W}}^{t}(s)=\left(W_{1}(s), W_{2}(s)\right) ; s \geq 0\right\}$ is defined such that

$$
\begin{aligned}
& \sup _{0 \leq s \leq 1} \|\left(\sum_{j=1}^{[m s]}\left(Z_{j, k}^{r} X_{j}-\mu \mu_{r, k}\right), \sum_{j=1}^{[m s]}\right. \\
& \left.\left(Z_{j, k}-k\right)\right)^{t}-\underline{\mathbf{W}}^{t}([m s]) \| \stackrel{\text { a.s. }}{=} o\left(m^{\frac{1}{4}}\right),
\end{aligned}
$$

where $E \underline{W}(\mathrm{~s})=0$, and

$$
E \underline{\mathbf{W}}(s) \underline{\mathbf{W}}^{t}(t)=\min (s, t)\left[\begin{array}{ll}
\sigma_{r, k}^{2} & r \mu \mu_{r, k} \\
r \mu \mu_{r, k} & k
\end{array}\right] .
$$

Theorem A follows from the results of Einmahl [13], Zaitsev [14] and Götze and Zaitsev [15].

The main result of this paper is the following Theorem.

Theorem 1. On some probability space, there exists a sequence of mean zero Gaussian processes $\Gamma_{m}(t, k, r, F), 0 \leq t \leq 1$ such that

$$
\begin{aligned}
& \sup _{0 \leq t \leq 1}\left|\alpha_{m}(t, k, r, F)-\Gamma_{m}(t, k, r, F)\right| \stackrel{P}{=} o\left(m^{-\frac{1}{4}}\right) \text {, (11) } \\
& \text { and where } \Gamma_{m}(t, k, r, F) \stackrel{D}{=} \Gamma(t, k, r, F) \text { for each } m \text {, }
\end{aligned}
$$

$$
E\{\Gamma(t, k, r, F) \Gamma(s, k, r, F)\}=(t \wedge s) \sigma_{r, k}^{2}-\frac{r^{2} \mu^{2} \mu_{r, k}^{2}}{k} t s .
$$

Theorem 1 follows from (6) and the following Theorem.

Theorem 2. On the probability space of Theorem A,

$$
\begin{gathered}
\sup _{0 \leq t \leq 1} \mid \beta_{m}(t, k, r, F)-m^{-\frac{1}{2}}\left\{W_{1}(m t)\right. \\
\left.-\frac{t r \mu \mu_{r, k}}{k} W_{2}(m)\right\} \mid \stackrel{\text { a.s. }}{=} o\left(m^{-\frac{1}{4}}\right),
\end{gathered}
$$

where $\underline{W}($.$) is as in (9).$

Proof of Theorem 2: We will only prove here the case when $E(X)=\mu \neq 0$. The case when $\mu=0$ is straightforward and can be looked at as a special case of the case $\mu \neq 0$. Note that

$$
\beta_{m}(t, k, r, F)=\frac{m^{\frac{1}{2}} k^{r} A_{m}(t)}{\left(\frac{1}{m} \sum_{i=1}^{m} z_{i, k}\right)^{r}}
$$

where

$$
\begin{aligned}
& A_{m}(t)=\frac{1}{m} \sum_{i=1}^{[m t]} Z_{j, k}^{r} X_{j}-t \mu \mu_{r, k} \frac{1}{k^{r}}\left(\frac{1}{m} \sum_{i=1}^{m} Z_{j, k}\right)^{r} \\
& =\frac{1}{m} \sum_{i=1}^{[m t]}\left(Z_{j, k}^{r} X_{j}-\mu \mu_{r, k}\right)+\mu \mu_{r, k} \frac{([m t]-m t)}{m}
\end{aligned}
$$




$$
+\mu \mu_{r, k} t-t \mu \mu_{r, k} \frac{1}{k^{r}}\left(\frac{1}{m} \sum_{i=1}^{m}\left(Z_{j, k}-k\right)+k\right)^{r} .
$$

It is clear that, uniformly in $t, 0 \leq t \leq 1$,

$$
\frac{|[m t]-m t|}{m}<\frac{1}{m}
$$

By (9), (15) and (16) we have, uniformly in $t, 0 \leq t \leq 1$,

$A_{m}(t) \stackrel{\text { a.s. }}{=} \frac{1}{m} W_{1}(m t)+\frac{1}{m}\left(W_{1}([m t])-W_{1}(m t)\right)+O\left(\frac{1}{m}\right)+$

$\mu \mu_{r, k} t-t \mu \mu_{r, k}\left(1+\frac{1}{m k} W_{2}(m)+o\left(m^{-\frac{3}{4}}\right)\right)^{r}+o\left(m^{-\frac{3}{4}}\right)$.

By Lemma 1.1.1 of Csörgö and Révész [17] we have, uniformly in $t, 0 \leq t \leq 1$,

$$
\frac{1}{m}\left|W_{1}([m t])-W_{1}(m t)\right| \stackrel{\text { a.s. }}{=} O\left(\frac{1}{m} \sqrt{\log m}\right) .
$$

By (17) and (18) we have, uniformly in $t, 0 \leq t \leq 1$,

$$
\begin{aligned}
& A_{m}(t) \stackrel{\text { a.s. }}{=} \frac{1}{m} W_{1}(m t)+O\left(\frac{1}{m} \sqrt{\log m}\right)+\mu \mu_{r, k} t \\
& -t \mu \mu_{r, k}\left(1+\frac{1}{m k} W_{2}(m)+o\left(m^{-\frac{3}{4}}\right)\right)^{r}+o\left(m^{-\frac{3}{4}}\right) \\
& \stackrel{\text { a.s. }}{=} \frac{1}{m} W_{1}(m t)-\frac{\operatorname{tr} \mu \mu_{r, k}}{m k} W_{2}(m)+o\left(m^{-\frac{3}{4}}\right) .
\end{aligned}
$$

By the LIL

$$
\begin{gathered}
\left(\frac{1}{m} \sum_{i=1}^{m} Z_{i, k}\right)^{r} \stackrel{\text { a.s. }}{=}\left(k+O\left(m^{-\frac{1}{2}} \sqrt{\log \log m}\right)\right)^{r} \\
\stackrel{\text { a.s. }}{=} k^{r}+O\left(m^{-\frac{1}{2}} \sqrt{\log \log m}\right) .
\end{gathered}
$$

By (14), (19) and (20) we have, uniformly in $t, 0 \leq t \leq 1$,

$$
\begin{gathered}
\beta_{m}(t, k, r, F) \stackrel{\text { a.s. }}{=} \frac{m^{\frac{1}{2}} k^{r}}{k^{r}+o\left(m^{-\frac{1}{2}} \sqrt{\log \log m}\right)}\left\{\frac{1}{m} W_{1}(m t)-\right. \\
\left.\frac{t r \mu \mu_{r, k}}{m k} W_{2}(m)+o\left(m^{-\frac{3}{4}}\right)\right\} \\
\stackrel{\text { a.s. }}{=} m^{-\frac{1}{2}}\left\{W_{1}(m t)-\frac{t r \mu \mu_{r, k}}{k} W_{2}(m)\right\}+o\left(m^{-\frac{1}{4}}\right) .
\end{gathered}
$$

This proves (13).

Corollary 1. By (4), (8) and (12),

$$
\Gamma(\cdot, k, r, F) \stackrel{D}{=} \lambda_{r, k} W(\cdot)+\frac{r \mu \Gamma(r+k)}{\sqrt{k} \Gamma(k)} B(\cdot)
$$

where

$$
\lambda_{r, k}^{2}=\frac{\Gamma(2 r+k)}{\Gamma(k)} \sigma^{2}+\mu^{2}\left\{\frac{\Gamma(2 r+k)}{\Gamma(k)}-\frac{\left(r^{2}+k\right) \Gamma^{2}(r+k)}{k \Gamma^{2}(k)}\right\},
$$

$\mathrm{W}($.$) is a Wiener process, \mathrm{B}($.$) is a Brownian bridge and$ $\mathrm{W}($.$) and \mathrm{B}($.$) are independent.$

Corollary 2. By (11) and (21) we have, as $\mathrm{m} \rightarrow \infty$,

$$
\alpha_{m}(\cdot, k, r, F) \stackrel{D}{\rightarrow} \Gamma(\cdot, k, r, F) \stackrel{D}{=} \lambda_{r, k} W(\cdot)+\frac{r \mu \Gamma(r+k)}{\sqrt{k} \Gamma(k)} B(\cdot)
$$

and, in particular,

$$
\alpha_{m}(1, k, r, F) \stackrel{D}{\rightarrow} N\left(0, \lambda_{r, k}^{2}\right)
$$

Some special cases of (22) and (23) are given . For $\mathrm{r}=1$ and $\mathrm{k} \geq 1$,

$$
\Gamma(\cdot, k, 1, F)=\bar{\sigma} \quad \sqrt{k(k+1)} W(\cdot)+\mu \sqrt{k} B(\cdot)
$$

and

$$
\begin{gathered}
\alpha_{m}(1, k, 1, F) \stackrel{D}{\rightarrow} N\left(0, k(k+1) \sigma^{2}\right) . \text { For } r>0 \text { and } k=1, \\
\Gamma(\cdot, 1, r, F) \stackrel{D}{=} \lambda_{r, 1} W(\cdot)+r \mu \Gamma(r+1) B(\cdot)
\end{gathered}
$$

and

$$
\alpha_{m}(1,1, r, F) \stackrel{D}{\rightarrow} N\left(0, \lambda_{r, 1}^{2}\right)
$$

where

$$
\lambda_{r, 1}^{2}=\sigma^{2} \Gamma(2 r+1)+\mu^{2}\left\{\Gamma(2 r+1)-\left(1+r^{2}\right) \Gamma^{2}(r+1)\right\} .
$$

\section{RESULTS}

In this section, we will use the same notation of Section 1

\subsection{The scaled sum case}

Define

$$
T_{m, 1}(t, k, r, F)= \begin{cases}\frac{1}{\sum_{j=1}^{m} X_{j}} \sum_{i=1}^{[m t]} R_{i, k}^{r} X_{i}, & \frac{1}{m} \leq t \leq 1 \\ 0, & 0 \leq t<\frac{1}{m}\end{cases}
$$

and

$$
\gamma_{m, 1}(t, k, r, F)= \begin{cases}m^{\frac{1}{2}}\left\{\frac{k^{r} m^{r}}{\mu_{r, k}} T_{m, 1}(t, k, r, F)-t\right\}, & \frac{1}{m} \leq t \leq 1 \\ 0, & 0 \leq t<\frac{1}{m} .\end{cases}
$$

We can prove that

$$
\gamma_{m, 1}(t, k, r, F) \stackrel{D}{\rightarrow} \gamma_{1}(t, k, r, F),
$$

where

$$
\gamma_{1}(t, k, r, F)=\frac{1}{\mu \mu_{r, k}} W_{1}(t)-\frac{r}{k} t W_{2}(1)-\frac{1}{\mu} t W_{3}(1),
$$

$\left(\mathrm{W}_{1}(.), \mathrm{W}_{2}(.), \mathrm{W}_{3}(.)\right)^{\mathrm{t}}$ is a mean zero Gaussian vector with covariance $(t \Lambda s) \sum_{1}$ and

$$
\Sigma_{1}=\left[\begin{array}{lll}
\sigma_{r, k}^{2} & r \mu \mu_{r, k} & \sigma^{2} \mu_{r, k} \\
r \mu \mu_{r, k} & k & 0 \\
\sigma^{2} \mu_{r, k} & 0 & \sigma^{2}
\end{array}\right]
$$

Let

$$
\delta_{r, k}^{2}=\left(\frac{\mu_{2 r, k}}{\mu_{r, k}^{2}}-1\right)\left(\frac{\sigma^{2}}{\mu^{2}}+1\right)-\frac{r^{2}}{k}
$$

We can show that 


$$
\gamma_{1}(t, k, r, F) \stackrel{D}{=} \delta_{r, k} W(t)+\sqrt{\frac{r^{2}}{k}+\frac{\sigma^{2}}{\mu^{2}}} B(t),
$$

where $W($.$) is a Brownian Motion and B($.$) is a Brownian$ bridge and $W($.$) and B($.$) are independent. Consequently,$

$$
\gamma_{m, 1}(1, k, r, F) \stackrel{D}{\rightarrow} N\left(0, \delta_{r, k}^{2}\right)
$$

When $r=1, k \geq 1$

$$
\delta_{1, k}^{2}=\frac{\sigma^{2}}{k \mu^{2}}
$$

When $r>0, k=1$

$$
\delta_{r, 1}^{2}=\left(\frac{\Gamma(2 r+1)}{\Gamma^{2}(r+1)}-1\right)\left(\frac{\sigma^{2}}{\mu^{2}}+1\right)-r^{2} .
$$

\subsection{The Centered Sum Process}

Let $\bar{X}=\frac{1}{m} \sum_{j=1}^{m} X_{j} \quad$ and define

$$
T_{m, 2}(t, k, r, F)= \begin{cases}\sum_{i=1}^{[m t]} R_{i, k}^{r}\left(X_{i}-\bar{X}\right), & \frac{1}{m} \leq t \leq 1 \\ 0, & 0 \leq t<\frac{1}{m}\end{cases}
$$

and

$$
\gamma_{m, 2}(t, k, r, F)= \begin{cases}k^{r} m^{r-\frac{1}{2}} T_{m, 2}(t, k, r, F), & \frac{1}{m} \leq t \leq 1 \\ 0, & 0 \leq t<\frac{1}{m}\end{cases}
$$

We can prove that

$$
\gamma_{m, 2}(t, k, r, F) \stackrel{D}{\rightarrow} \gamma_{2}(t, k, r, F),
$$

where

$$
\gamma_{2}(t, k, r, F)=W_{1}(t)-\mu W_{2}(t)-\mu_{r, k} t W_{3}(1),
$$

$\left(W_{1}(.), W_{2}(.), W_{3}(.)\right)^{\mathrm{t}}$ is a mean zero Gaussian vector with covariance $(t \Lambda s) \sum_{2}$ and

$$
\Sigma_{2}=\left[\begin{array}{lll}
\sigma_{r, k}^{2} & \mu\left(\mu_{2 r, k}-\mu_{r, k}^{2}\right) & \sigma^{2} \mu_{r, k} \\
\mu\left(\mu_{2 r, k}-\mu_{r, k}^{2}\right) & \mu_{2 r, k}-\mu_{r, k}^{2} & 0 \\
\sigma^{2} \mu_{r, k} & 0 & \sigma^{2}
\end{array}\right]
$$

We can show that

$$
\gamma_{2}(t, k, r, F) \stackrel{D}{=} \sigma\left\{\sqrt{\mu_{2 r, k}-\mu_{r, k}^{2}} W(t)+\mu_{r, k} B(t)\right\},
$$

where $W($.$) is a Brownian Motion and B($.$) is a Brownian$ bridge and $W($.) and $B($.$) are independent. Consequently,$

$$
\gamma_{m, 2}(1, k, r, F) \stackrel{D}{\rightarrow} N\left(0, \sigma^{2}\left(\mu_{2 r, k}-\mu_{r, k}^{2}\right)\right) .
$$

When $r=1, k \geq 1$

$$
\gamma_{m, 2}(1, k, r, F) \stackrel{D}{\rightarrow} N\left(0, k \sigma^{2}\right) .
$$

When $r>0, k=1$

$$
\gamma_{m, 2}(1, k, r, F) \stackrel{D}{\rightarrow} N\left(0, \sigma^{2}\left(\Gamma(2 r+1)-\Gamma^{2}(r+1)\right)\right) .
$$

\subsection{The Renewal Process}

For simplicity, we will consider the case of $r=1$. Define

$$
\begin{aligned}
& S_{m}^{*}(t)= \begin{cases}\frac{1}{\mu} \sum_{i=1}^{[m t]} R_{i, k} X_{i}, & \frac{1}{m} \leq t \leq 1 \\
0, & 0 \leq t<\frac{1}{m}\end{cases} \\
& T_{m}^{*}(t)= \begin{cases}\frac{1}{\mu} \sum_{i=1}^{[m t]} Z_{i, k} X_{i} /\left(\sum_{i=1}^{m} Z_{i, k}\right), & \frac{1}{m} \leq t \leq 1 \\
0, & 0 \leq t<\frac{1}{m}\end{cases} \\
& N_{m}(t)=\inf \left\{u: S_{m}^{*}(u)>t\right\}, \\
& M_{m}(t)=\inf \left\{u: T_{m}^{*}(u)>t\right\}, \\
& \alpha_{m}^{*}(t)= \begin{cases}m^{\frac{1}{2}} k \mu\left\{S_{m}^{*}(t)-t\right\}, & \frac{1}{m} \leq t \leq 1 \\
0, & 0 \leq t<\frac{1}{m}\end{cases} \\
& \beta_{m}^{*}(t)= \begin{cases}m^{\frac{1}{2}} k \mu\left\{T_{m}^{*}(t)-t\right\}, & \frac{1}{m} \leq t \leq 1 \\
0, & 0 \leq t<\frac{1}{m}\end{cases} \\
& \eta_{m}(t)=m^{\frac{1}{2}} k \mu\left\{t-N_{m}(t)\right\}
\end{aligned}
$$

and

$$
\xi_{m}(t)=m^{\frac{1}{2}} k \mu\left\{t-M_{m}(t)\right\}
$$

By (5), for each $m$

$$
\alpha_{m}^{*}(\cdot) \stackrel{D}{=} \beta_{m}^{*}(\cdot) \text { and } \eta_{m}(\cdot) \stackrel{D}{=} \xi_{m}(\cdot) .
$$

Note that (see (3))

$$
\alpha_{m}^{*}(\cdot)=\alpha_{m}(\cdot, k, 1, F)
$$

and hence, by Theorem 1

$$
\sup _{0 \leq t \leq 1}\left|\alpha_{m}^{*}(t)-\Gamma_{m}(t, k, 1, F)\right| \stackrel{P}{=} o\left(m^{-\frac{1}{4}}\right),
$$

where $\Gamma_{\mathrm{m}}(., k, 1, F)$ is as in (11).

Theorem 3. On the probability space of Theorem A,

$$
\sup _{0 \leq t \leq 1}\left|\eta_{m}(t)-\Gamma_{m}(t)\right| \stackrel{P}{=} o\left(m^{-\frac{1}{4}}(\log m \log \log m)^{\frac{1}{2}}\right),
$$

where

$$
\Gamma_{m}(t)=m^{-\frac{1}{2}}\left\{W_{1}(m t)-t \mu W_{2}(m)\right\}
$$

and $\underline{W}($.$) is as in (9).$ 
Theorem 3 follows directly from (24) and the following Theorem.

Theorem 4. On the probability space of Theorem A,

$$
\sup _{0 \leq t \leq 1}\left|\xi_{m}(t)-\Gamma_{m}(t)\right| \stackrel{\text { a.s. }}{=} O\left(m^{-\frac{1}{4}}(\log m \log \log m)^{\frac{1}{2}}\right)
$$

where $\Gamma_{m}(t)$ is as in (25).

Proof: By (7),

$$
\beta_{m}^{*}(\cdot)=\beta_{m}(\cdot, k, 1, F)
$$

Note that

$$
\xi_{m}(t)=\beta_{m}^{*}\left(M_{m}(t)\right)-m^{\frac{1}{2}} k \mu\left\{T_{m}\left(M_{m}(t)\right)-t\right\}
$$

Hence

$$
\sup _{0 \leq t \leq 1}\left|\xi_{m}(t)-\Gamma_{m}(t)\right| \leq E_{m 1}+E_{m 2}+E_{m 3}
$$

where

$$
\begin{gathered}
E_{m 1}=\sup _{0 \leq t \leq 1}\left|\beta_{m}^{*}\left(M_{m}(t)\right)-\Gamma_{m}\left(M_{m}(t)\right)\right|, \\
E_{m 2}=m^{\frac{1}{2}} k \mu \sup _{0 \leq t \leq 1}\left|T_{m}\left(M_{m}(t)\right)-t\right|
\end{gathered}
$$

and

$$
E_{m 3}=\sup _{0 \leq t \leq 1}\left|\Gamma_{m}\left(M_{m}(t)\right)-\Gamma_{m}(t)\right|
$$

By Theorem 2 and the LIL for Wiener processes,

$$
E_{m 1} \stackrel{\text { a.s. }}{=} o\left(m^{-\frac{1}{4}}\right)
$$

and

$$
\sup _{0 \leq t \leq 1}\left|T_{m}(t)-t\right| \stackrel{\text { a.s. }}{=} O\left(\sqrt{m^{-1} \log \log m}\right)
$$

By a Lemma of Horváth [18]

$$
\sup _{0 \leq t \leq 1}\left|M_{m}(t)-t\right| \leq \sup _{0 \leq t \leq 1}\left|T_{m}(t)-t\right|
$$

and hence

$$
\sup _{0 \leq t \leq 1}\left|M_{m}(t)-t\right| \stackrel{\text { a.s. }}{=} O\left(\sqrt{m^{-1} \log \log m}\right)
$$

By the proof of Step 5 of Horváth [18] and Theorem 2 we can show that

$$
E_{m 2} \stackrel{a . s .}{=} O\left(m^{-\frac{1}{4}} \log m\right)
$$

As to $E_{\mathrm{m} 3}$,

$$
E_{m 3} \leq E_{m 31}+E_{m 32}
$$

where

$$
E_{m 31}=\sup \left|W_{1}\left(M_{m}(t)\right)-W_{1}(t)\right|
$$

and

$$
E_{m 32}=m^{-\frac{1}{2}} \mu\left|W_{2}(m)\right| \sup \left|M_{m}(t)-t\right| .
$$

By (28) and Lemma 1.1.1 of Csörgö and Révész [17] we have, uniformly in $t, 0 \leq t \leq 1$,

$$
\begin{aligned}
E_{m 31}= & \sup \left|W_{1}\left(t+\left(M_{m}(t)-t\right)\right)-W_{1}(t)\right| \\
\stackrel{\text { a.s. }}{=} & \sup _{0 \leq h \leq m^{-\frac{1}{2}}(\log \log m)^{\frac{1}{2}}}\left|W_{1}(t+h)-W_{1}(t)\right| \\
& \stackrel{\text { a.s. }}{=} O\left(m^{-\frac{1}{4}} \sqrt{\log m \log \log m}\right) .
\end{aligned}
$$

By (28) and the LIL for Wiener processes,

$$
E_{m 32} \stackrel{\text { a.s. }}{=} O\left(m^{-\frac{1}{2}} \log \log m\right)
$$

By (30)-(32),

$$
E_{m 3} \stackrel{\text { a.s. }}{=} O\left(m^{-\frac{1}{4}} \sqrt{\log m \log \log m}\right) .
$$

By (26)-(33) we obtain Theorem 4.

\section{THE RANDOM VECTOR CASE}

Let $\underline{X}_{1}, \underline{X}_{2}, \ldots$ be iid random vectors with $E\left(\underline{X}_{i}\right)=\underline{\mu}=\left(\mu_{1}, \mu_{2}, \cdots, \mu_{p}\right)^{t}$ and $\operatorname{Var}\left(\underline{X}_{i}\right)=\sum=\left[\sigma_{i j}\right]$.

Assume that the $U_{i} s$ and the $R_{i, k} s$ are same as in Section 1 and are independent of $\underline{X}_{1}, \underline{X}_{2}, \ldots$ Define

$$
\underline{S}_{m}(t, k, r, F)= \begin{cases}\sum_{i=1}^{[m t]} R_{i, k}^{r} \underline{X}_{i}, & \frac{1}{m} \leq t \leq 1 \\ 0, & 0 \leq t<\frac{1}{m}\end{cases}
$$

and

$$
\underline{\alpha}_{m}(t, k, r, F)=\left\{\begin{array}{c}
0, \quad 0 \leq t<\frac{1}{m} . \\
m^{\frac{1}{2}}\left\{k^{r} m^{r-1} \underline{S}_{m}(t, k, r, F)\right. \\
\left.-t \mu_{r, k} \underline{\mu}\right\}, \quad \frac{1}{m} \leq t \leq 1
\end{array}\right.
$$

Theorem 5 is a generalization of Theorem 1 .

Theorem 5. On some probability space, there exists a mean zero sequence of Gaussian processes $\left\{\Gamma_{m}(t, k, r, F), 0 \leq t \leq 1\right\}$ such that 


$$
\sup _{0 \leq t \leq 1}\left\|\underline{\alpha}_{m}(t, k, r, F)-\underline{\Gamma}_{m}(t, k, r, F)\right\| \stackrel{P}{=} o\left(m^{-\frac{1}{4}}\right)
$$

where, for each $\mathrm{m}$,

$$
\begin{gathered}
\Gamma_{m}(\cdot, k, r, F) \stackrel{D}{=} \underline{\Gamma}(\cdot, k, r, F), \\
E \underline{\Gamma}(s, k, r, F) \underline{\Gamma}^{t}(t, k, r, F)= \\
(t \wedge s) \sum_{r, k}^{(1)}-\frac{r^{2} \Gamma^{2}(r+k)}{k \Gamma^{2}(k)} t s \underline{\mu \mu^{t}}
\end{gathered}
$$

and

$$
\sum_{r, k}^{(1)}=\frac{\Gamma(2 r+k)}{\Gamma(k)} \sum+\left\{\frac{\Gamma(2 r+k)}{\Gamma(k)}-\frac{\Gamma^{2}(r+k)}{\Gamma^{2}(k)}\right\} \underline{\mu \mu^{t}} .
$$

Corollary 1 *. By (11) and (21) we have, as $\mathrm{m} \rightarrow \infty$,

$$
\underline{\alpha}_{m}(\cdot, k, r, F) \stackrel{D}{\rightarrow} \underline{\Gamma}(\cdot, k, r, F)
$$

and, in particular,

$$
\underline{\alpha}_{m}(1, k, r, F) \stackrel{D}{\rightarrow} M V N\left(\underline{0}, \Sigma_{r, k}^{(2)}\right),
$$

where

$$
\sum_{r, k}^{(2)}=\frac{\Gamma(2 r+k)}{\Gamma(k)} \sum+\left\{\frac{\Gamma(2 r+k)}{\Gamma(k)}-\frac{\left(k+r^{2}\right) \Gamma^{2}(r+k)}{k \Gamma^{2}(k)}\right\} \underline{\mu \mu^{t}} .
$$

Particular cases of Corollary $1 *$ are given next.

For $r=1$ and $k \geq 1$,

$$
\begin{gathered}
E \underline{\Gamma}(s, k, 1, F) \underline{\Gamma}^{t}(t, k, 1, F)= \\
(t \wedge s) \sum_{1, k}^{(1)}-t s k \underline{\mu \mu^{t}}, \\
\sum_{1, k}^{(1)}=k(k+1) \sum+k \underline{\mu \mu}{ }^{t}
\end{gathered}
$$

and

$$
\underline{\Gamma}(1, k, 1, F) \stackrel{D}{=} \operatorname{MVN}\left(\underline{0}, k(k+1) \sum\right) .
$$

For $r>0$ and $k=1$,

$$
\begin{gathered}
E \underline{\Gamma}(s, 1, r, F) \underline{\Gamma}^{t}(t, 1, r, F)= \\
(t \wedge s) \sum_{r, 1}^{(1)}-t s r^{2} \Gamma^{2}(r+1) \underline{\mu \mu^{t}}, \\
\sum_{r, 1}^{(1)}=\Gamma(2 r+1) \Sigma+ \\
\left\{\Gamma(2 r+1)-\Gamma^{2}(r+1)\right\} \underline{\mu \mu}{ }^{t}
\end{gathered}
$$

and

$$
\underline{\Gamma}(1,1, r, F) \stackrel{D}{=} \operatorname{MVN}\left(\underline{0}, \Sigma^{*}\right)
$$

where

$$
\begin{aligned}
\sum^{*}= & \Gamma(2 r+1) \sum+\{\Gamma(2 r+1) \\
& \left.-\left(1+r^{2}\right) \Gamma^{2}(r+1)\right\} \underline{\mu \mu} \underline{\mu}^{t}
\end{aligned}
$$

\section{CONCLUSION}

We proved the weak convergence of a stochastic process defined in terms of partial sums of randomly weighted powers of uniform spacings. The asymptotic results of several important generalizations and special cases are given.

\section{CONSENT FOR PUBLICATION}

Not applicable.

\section{AVAILABILITY OF DATA AND MATERIALS}

Not applicable.

\section{FUNDING}

None.

\section{CONFLICT OF INTEREST}

The author declare no conflict of interest, financial or otherwise.

\section{ACKNOWLEDGEMENTS}

Declared none.

\section{REFERENCES}

[1] W. Van Assche, "A random variable uniformly distributed between two independent random variables", Sankhya A, vol. 49, no. 2, pp. 207-211, 1987.

[2] N.L. Johnson, and S. Kotz, "Randomly weighted averages: Some aspects and extensions", Am. Stat., vol. 44, no. 3, pp. 245-249, 1990.

[3] A.R. Soltani, and H. Homei, "Weighted averages with random proportions that are jointly uniformly distributed over the unit simplex", Stat. Probab. Lett., vol. 79, no. 9, pp. 1215-1218, 2009. [http://dx.doi.org/10.1016/j.spl.2009.01.009]

[4] A.R. Soltani, and R. Roozegar, "On distribution of randomly ordered uniform incremental weighted averages: Divided difference approach", Stat. Probab. Lett., vol. 82, no. 5, pp. 1012-1020, 2012.

[http://dx.doi.org/10.1016/j.spl.2012.02.007]

[5] H.E. Kimball, "On the asymptotic distribution of the sum of powers of unit frequency differences", Ann. Math. Stat., vol. 21, no. 2, pp. 263-271, 1950.

[http://dx.doi.org/10.1214/aoms/1177729843]

[6] D.A. Darling, "On a class of problems related to the random division of an interval", Ann. Math. Stat., vol. 24, no. 2, pp. 239-253, 1953. [http://dx.doi.org/10.1214/aoms/1177729030]

[7] L. LeCam, "Un theoreme sur la division d'un intervalle par des points pris au hasard", Publ. Inst. Stat. Univ. Paris, vol. 7, no. 3/4, pp. 7-16, 1958 .

[8] J. Sethuraman, and J.S. Rao, "Weak convergence of empirical distribution functions of random variables subject to perturbations and 
scale factors", Ann. Stat., vol. 3, no. 2, pp. 299-313, 1975. [http://dx.doi.org/10.1214/aos/1176343058]

[9] J.A. Koziol, "A note on limiting distributions for spacings statistics", Z. Wahrsch. Verw. Gebiete, vol. 51, no. 1, pp. 55-62, 1980 [http://dx.doi.org/10.1007/BF00533817]

[10] E-E.A.A. Aly, "Some limit theorems for uniform and exponential spacings", Canad. J. Statist., vol. 11, no. 1, pp. 211-219, 1983.

[11] E-E.A.A. Aly, "Strong approximations of quadratic sums of uniform spacings", Can. J. Stat., vol. 16, no. 2, pp. 201-207, 1988. [http://dx.doi.org/10.2307/3314641]

[12] L. Breiman, Probability., Addison-Wesley: Reading, Massachusetts, 1968.

[13] U. Einmahl, "Extension of results of Komlós, Major and Tusnády to the multivariate case", J. Mult. Anal., vol. 28, no. 1, pp. 20-68, 1989.
[http://dx.doi.org/10.1016/0047-259X(89)90097-3]

[14] A.Yu. Zaitsev, "Multidimensional version of the results of Komlós, Major and Tusnády for vectors with finite exponential moments", ESAIM Probab. Stat., vol. 2, pp. 41-108, 1998.

[http://dx.doi.org/10.1051/ps:1998103]

[15] F. Götze, and A.Yu. Zaitsev, "Bounds for the rate of strong approximation in the multidimensional invariance principle", Theory Probab. Appl., vol. 53, no. 1, pp. 100-123, 2008.

[16] M. Csörgö, and L. Horváth, Weighted Approximations in Probability and Statistics., John Wiley and Sons: New York, 1993.

[17] M. Csörgö, and P. Revesz, P, Strong Approximations in Probability and Statistics., Academic Press: New York, 1981.

[18] L. Horváth, "Strong approximation of renewal processes", Stochastic Process. Appl., vol. 18, no. 1, pp. 127-138, 1984. [http://dx.doi.org/10.1016/0304-4149(84)90166-2]

\section{C) 2020 Emad-Eldin A. A. Aly.}

This is an open access article distributed under the terms of the Creative Commons Attribution 4.0 International Public License (CC-BY 4.0), a copy of which is available at: https://creativecommons.org/licenses/by/4.0/legalcode. This license permits unrestricted use, distribution, and reproduction in any medium, provided the original author and source are credited. 